\title{
Guided modes in arrays of metallic nanowires
}

\author{
C. G. Poulton, M. Schmidt, G. Pearce, G. Kakarantzas and P. St.J. Russell \\ Max-Planck Research Group (IOIP), University of Erlangen-Nuremberg, Guenther-Scharowsky-Str. 1, Erlangen, Germany \\ e-mail: cpoulton@optik.uni-erlangen.de \\ Abstract: We study numerically the formation of photonic band gaps and guided "defect" modes within two \\ dimensional arrays of metallic nanowires. Attenuations as low as $1.7 \mathrm{~dB} / \mathrm{cm}$ are predicted for silver wires at \\ $1550 \mathrm{~nm}$ wavelength.
}

(C)2007 Optical Society of America

OCIS Codes: (060.2310) Fiber Optics; (240.6680) Surface Plasmons; (999.9999) Photonic Crystal Fibres;

\section{Introduction}

Photonic crystal fibres usually achieve confinement of light using an array of air-holes within a silica matrix [1]. We examine here the guidance properties of PCF in which the air channels are replaced with metallic wires. The possibility of exploiting interactions between guided light and surface plasmon modes which can exist at the metaldielectric interface has led to structures such as these being proposed for use as sensors and fiber-integrated optoelectronic components $[2,3]$. The high contrast in the permittivity may lead to modes which are more resistant to bends and other structural deformations, and field enhancements at the metal-glass interfaces could be used to increase nonlinear interactions. Here we calculate the positions of the photonic band-gaps (PBGs) of a triangular lattice of nanowires, and calculate the effective index and attenuation of the guided modes resulting from removing a single wire.

\section{Band-structure calculations}

We first consider an infinite array of metallic wires in order to determine the conditions necessary to achieve guidance, namely, the existence of a band-gap below the light-line of the "host" material surrounding the wires. Because the frequency dependence of the material properties breaks the scale invariance of Maxwell's equations, it is instructive to consider a fixed operating wavelength and then examine a range of geometries. For these calculations we consider a hexagonal lattice of silver nanowires (pitch $\Lambda$, diameter d) embedded in a silica glass matrix. For a vacuum wavelength $\lambda_{0}=1.55 \mu \mathrm{m}$ we find that the permittivities $\varepsilon_{\mathrm{m}}$ of the metal and $\varepsilon_{\mathrm{s}}$ of silica are $\varepsilon_{\mathrm{m}}=$ -123.5 and $\varepsilon_{\mathrm{s}}=2.085$, where in order to arrive at a meaningful definition of the band-structure, we assume that the material absorption is negligible (it will be included later). We use the multipole method [4], which has the advantage that it is capable of accurately modelling arrays with highly contrasting optical properties.
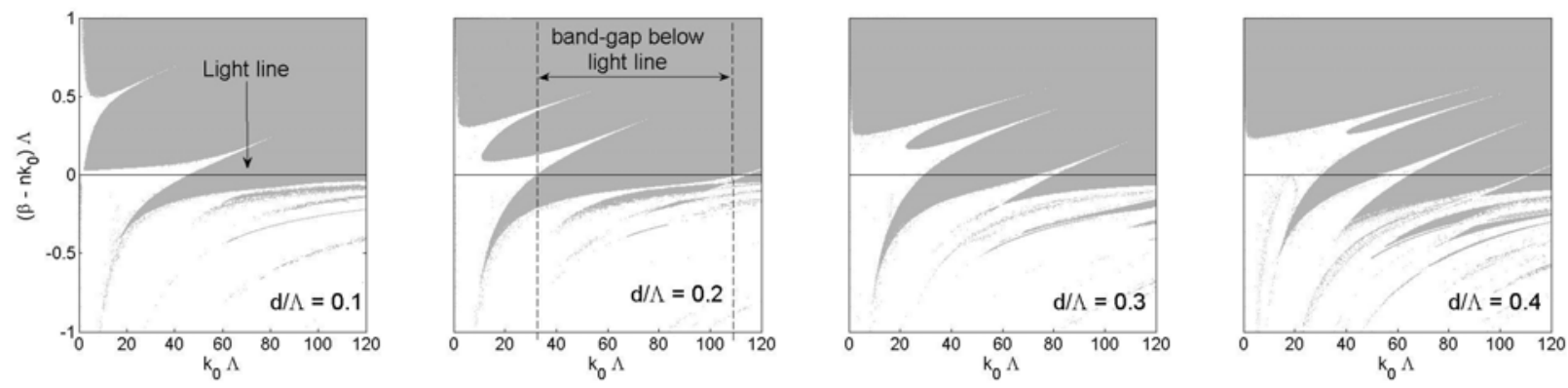

Figure 1: Presence of band-gaps (grey) as a function of normalised frequency $k \Lambda$, for different wire diameters d. All calculations are performed for the optical properties of silver and silica at $\lambda_{0}=1.55 \mu \mathrm{m}$. The coupled plasmon modes appear as resonances above the light line. Due to the strong interaction between the plasmon modes large normalised frequencies $\left(\mathrm{k}_{0} \Lambda>30\right)$ are necessary in order to establish a gap below the light line.

The position of the band-gaps as a function of normalised frequency for a number of different wire diameters is plotted in Figure 1. The plasmon modes appear as resonances above the light line. The existence of a PBG which can be used to guide a defect mode is dependent on the positions of the cut-offs of these plasmon modes, together with the effects of coupling between them, which result in a broadening of each resonance. The strongly coupled nature of the first two plasmon resonances means that they begin to interact significantly even above the light line, and so in general large values of $\mathrm{k}_{0} \Lambda(>\sim 30)$ are necessary in order to achieve a PBG. For the material properties given above, this choice of $\mathrm{k}_{0} \Lambda$ corresponds to a pitch of at least $8 \mu \mathrm{m}$ for all values of $\mathrm{d} / \Lambda$ between 0.05 and 0.55 . 


\section{CWC6.pdf}

\section{Guided mode calculations}

We now consider the case of a finite structure consisting of three rings of wires with a single wire removed from the centre of the array to form the guiding core (see Figure 2c). In contrast with the previous section, we consider the frequency dependence of the permittivities, and no longer neglect the material absorption. Using the multipole method we then calculate both the effective refractive index of the guided mode and the attenuation.
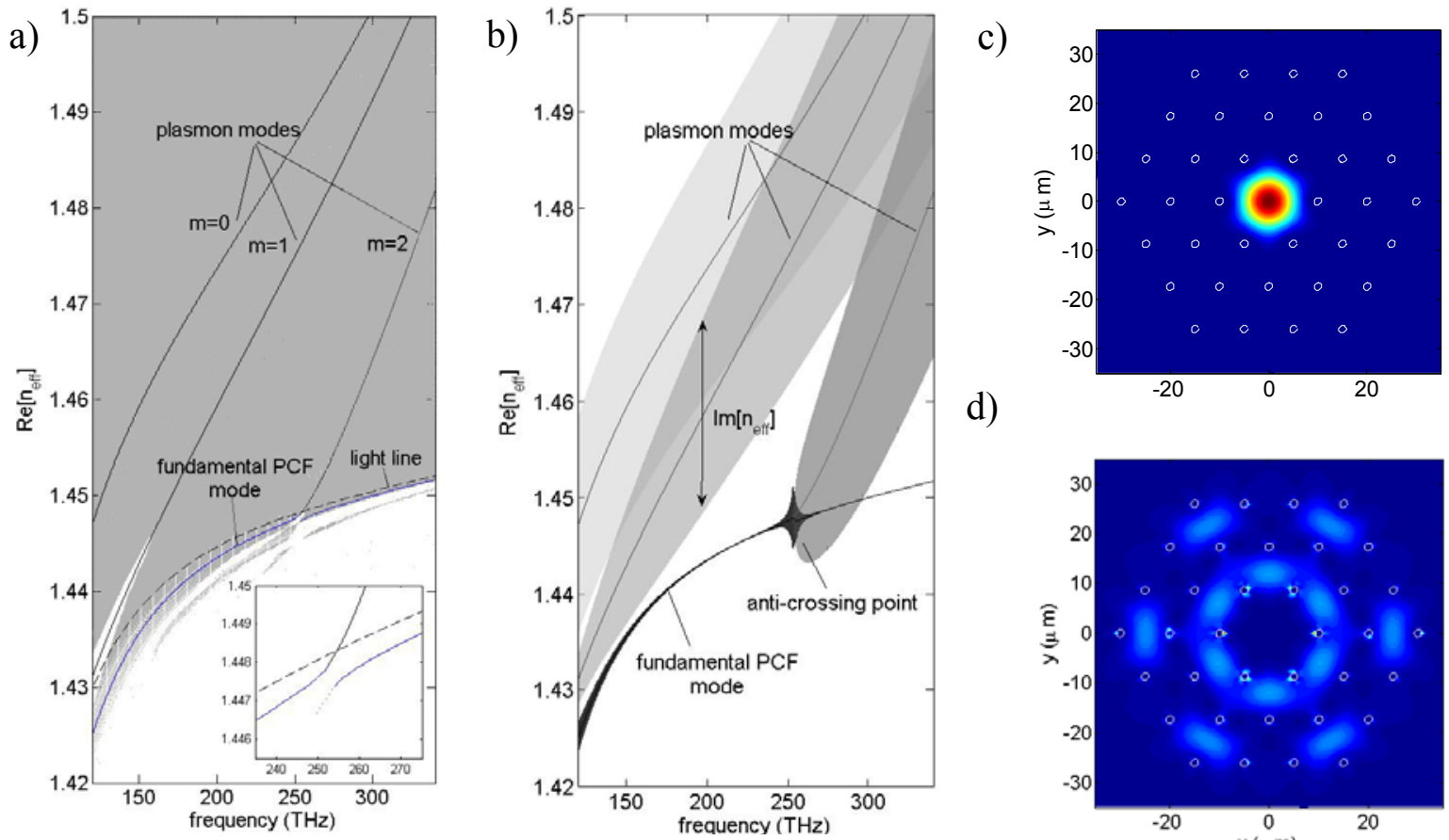

d)

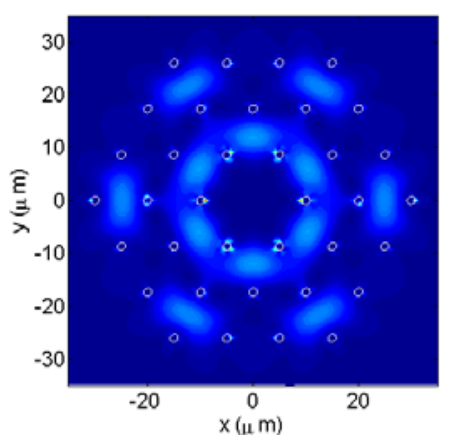

Figure 2: a) Fundamental guided mode and band-gaps (gray regions) of an array of nanowires consisting of silver cylinders in a silica matrix, with diameter $1.5 \mu \mathrm{m}$ and pitch $10 \mu \mathrm{m}$. The guided mode exists within the PBG below the light line (dashed), and exhibits an anti-crossing with the quadrupolar plasmon mode at around $v_{0}=253 \mathrm{THz}$ (inset). The dispersion relations for the plasmon modes of individual wires are plotted in black. b) Representation of dispersion and attenuation in the fundamental guided mode (black), as compared to the first three plasmon modes. The imaginary part of the effective index $\left(\operatorname{Im}\left[\mathrm{n}_{\mathrm{eff}}\right]\right)$ is depicted as the shaded region on either side of the mode itself, and shows the relative magnitude of the attenuation. For clarity $\operatorname{Im}\left[\mathrm{n}_{\mathrm{eff}}\right]$ has been multiplied by a factor of 50 . One can see a dramatic increase in the attenuation of the defect mode at the anti-crossing point. c) Real part of the Poynting vector of the guided mode at frequency $v_{0}=193.5 \mathrm{THz}\left(\lambda_{0}=1.55 \mu \mathrm{m}\right)$. d) Real part of the Poynting vector of the guided mode near the anti-crossing $\left(v_{0}=240 \mathrm{THz}, \lambda_{0}=1.25 \mu \mathrm{m}\right)$.

To achieve a PBG at $\lambda_{0}=1.55 \mu \mathrm{m}$, we choose a structure with a pitch of $10 \mu \mathrm{m}$ and wire diameter $d$ of $1.5 \mu \mathrm{m}$. The effective refractive index $n_{\text {eff }}$ of the fundamental guided mode is shown in Figure $2 a$, together with the PBGs for this geometry. The fundamental mode can be seen to exist within the gap below the light line, and undergoes an anti-crossing with the second order plasmon mode at a frequency of $v_{0}=253 \mathrm{THz}$. The attenuation of this mode can be calculated from the imaginary part of the effective index; this is depicted graphically in Figure $2 b$. We can see that the attenuation for this guided mode is much smaller than that of the plasmon modes existing above the light line; this is because most of the light of the guided mode is confined to the non-lossy region. For the mode at $\lambda_{0}=1.55 \mu \mathrm{m}$ (shown in Figure $2 \mathrm{c}$ ) the attenuation has been calculated to be $1.7 \mathrm{~dB} / \mathrm{cm}$, which is relatively small when compared with other metallic waveguides at communications frequencies [5]. One can also see that the anticrossing is accompanied by a substantial increase in attenuation. In Figure $2 \mathrm{~d}$ we see that the mode extends strongly into the array near the resonance maximum, and an examination (not shown here) of the z-component of the electric field around one of the cylinders reveals a strong quadrupolar component. We thus observe that on one side of the anti-crossing the fundamental mode undergoes a smooth transition to an array of surface-plasmon resonances, while on the other side the fundamental mode becomes a sum of Bloch modes with appropriate symmetry.

In conclusion, two-dimensional photonic crystal arrays of metallic nanowires display photonic band gaps, and are interesting and potentially useful as optical waveguides.

\section{References}

[1] P. St.J. Russell, "Photonic Crystal Fibers", Science 299, 358 - 362 (2003).

[2] P. J. A. Sazio et al., "Microstructured optical fibers as high-pressure microfluidic reactors," Science 311, 1583-1586 (2006).

[3] B.T. Kuhlmey et al., "Multipole analysis of photonic crystal fibers with coated inclusions", Optics Express 14, 10851-10864 (2006).

[4] T. P. White, et al., "Multipole method for microstructured optical fibers. I. Formulation", JOSA B 19, 2322-2330 (2002).

[5] E. Ozbay, "Plasmonics: Merging photonics and electronics at nanoscale dimensions", Science 311, 189-193 (2006). 